\title{
Effect of Tilt Angle of Tablet on Pen-based Input Operation Based on Fitts' Law
}

\author{
Dongxing Bao \\ College of Electronic Engineering \\ Heilongjiang University \\ Harbin, Heilongjiang, China \\ bdx2008@yahoo.com.cn
}

\author{
Yizhong Xin and Xiangshi Ren \\ School of Information \\ Kochi University of Technology \\ Kamishi, Kochi, Japan \\ ren.xiangshi@kochi-tech.ac.jp
}

\begin{abstract}
Current studies on tablets and digital pens mainly focus on the modalities of input in the field of HCI (human computer interaction). However, the effect of the changes of environmental parameters on pen-based input operation, such as tilt angle of tablet, has rarely been studied. This paper mainly investigates 1) how pen-based input operation is affected while changing tilt angle of tablet 2) at which tilt angle of tablet, can user do pen-based input operation with comfort, quickness, and accuracy. The conclusions can be got based on the results from the experiments and questionnaire. In order to compare penbased input operation among different tilt angle of tablet $(0,15$, 30, 45 and 60 degrees), two experiments of 1-dimensional and 2dimensional pointing tasks based on Fitts' law were conducted. Results show that when tilt angle of tablet is $\mathbf{3 0}$ degrees, it is most suitable for user to do pen-based input operation.
\end{abstract}

Index Terms - HCI, tilt angle of tablet, pen-based input operation, Fitts' law.

\section{INTRODUCTION}

Pointing devices used for human-computer interaction can be classified as direct input device and indirect input device. A direct input device can be used to do input by pointing on the touch screen directly. When using a indirect input device, a cursor on the screen is controlled by it to implement input operation. Mouse is the most representative one among indirect input devices (move it on the table to control a cursor to point to a spot on the screen). Pen mainly belongs to direct input device (touch the desired spot on the touch screen with it directly). Whereas, when pen is used to do input by controlling a cursor on the screen via a tablet, it belongs to indirect input device.

When pen is used as direct input device, it can be felt more directly and can be more accuracy compared with mouse. Moreover, no matter it is used as direct or indirect input device, pen mimics the shape of writing appliances, such as pencil and writing brush, and how to hold and use it is being familiar with since childhood by user.

Nowadays, pen can be applied to various devices with touch screens, such as LCD (liquid crystal display) tablets, tablet PCs, PDAs (personal digital assistant), intelligent mobile phones and tablets, etc. When information from pen are used by the interface, it is necessary to insure that the good performance can be got via design while environment changes. Therefore, investigations are needed to get the effect on pen-based input operation caused by the changes of various operating environments. Based on the results of experiments designed in various testing environments carefully, the changes of performance can be compared.

This paper focuses on the effect on pen-based input operation while one of the environmental conditions, i.e. the tilt angle of tablet, changes. The most useful usage of pen is doing direct input operation on the tablet. The performance can be affected by many factors when pen is used as direct input device including device and individual difference. However, the tilt angle of tablet is the main factor to affect pen-based input operation, because it can often be changed especially during the process of input operation. Thus further study on effect of tilt angle of tablet on pen based input operation is necessary. Based on the study, we can also find that at which tilt angle of tablet it is more suitable for user to do pen-based input operation.

In this study, a pen was used as direct input device to a LCD tablet. The range of the changes of tilt angle of tablet was from 0 to 60 degrees, and 5 kinds of tilt angles of tablet $(0,15,30,45$ and 60 degrees) were investigated. 1dimensional and 2-dimensional pointing tasks were done in each condition to get the effect on pen-based input operation in different tilt angle of tablet. Based on the results of the experiments and questionnaire, we can find the appropriate tilt angle of tablet for pen-based input operation. The results from this paper can be a reference to aid application and device of pen-based input interface.

\section{RELATED WORK}

Nowadays, Studies on tablet mainly focus on pressure sensitive of pen-based interface. Early investigation of the use of pressure in user interface was done by Herot [18] to test the ability of the human finger in controlling a cursor's position and speed on the touch screen. Buxton et al. [19] explored touch-sensitive tablet input and presented an example of a painting application that employs continuous pressure sensing to control the width of the tool being used. A pressure sensitive widget [20] could provide meaningful feedbacks to make user aware of the pen's pressure. We can also find studies on the use of pressure at the user interface in [21], [22], [23].

Works on tilting screen mainly investigate text input method, such as TiltType [1] and TiltText [2]. TiltType implemented by Partridge et al. was a watch-sized text entry device and TiltText devised by D. Wigdor and R. Balakrishnan was a new technique for entering text into a mobile phone. 
These two devices used the combination of a button press and tilting of the device to determine the desired letter. To enter a character, the user tilted the device and pressed one or more buttons. The character chosen depended on the button pressed, the direction of tilt, and the angle of tilt. TiltText differed from TiltType in the keypad used and sensing algorithms. The design of these two devices was inspired by Unigesture [3], enabled one-handed text-entry on PDA or cellphone-sized devices. Unigesture did not use buttons. Instead, a tilting gesture was mapped to a group of characters, and a sequence of gestures was mapped to a specific word by T9-style dictionary lookup. Unigesture was inspired by nontext-entry accelerometer-based user interfaces had been done by Rekimoto [4], Harrison et al. [5], and Levin and Yarin [6].

Currently, the study on pen mainly focused on inherent input modalities of pen to enhance pen-based input, such as Pressure, Tilt, Azimuth, and Rolling (see Fig.1). G. Ramos et al. [7] investigated human ability to use stylus pressure to perform discrete target acquisition tasks and found that dividing pressure space into 6 levels was optimal. X. Bi et al. [8] investigated incidental pen rolling made by users when performing regular drawing and writing tasks, as well as users' ability to control intentional rolling and presented an exploration of the design space of rolling-based interaction techniques. F. Tian et al. [9] utilized pen tilt for interaction: matching cursor shape to tilt angles could improve performance by enhancing stimulus-response compatibility. M. Oshita [10] presented a pen-based intuitive interface to control a virtual human figure interactively, and in this design, the position, pressure, and tilt of the pen was used to make the figure perform various motions.

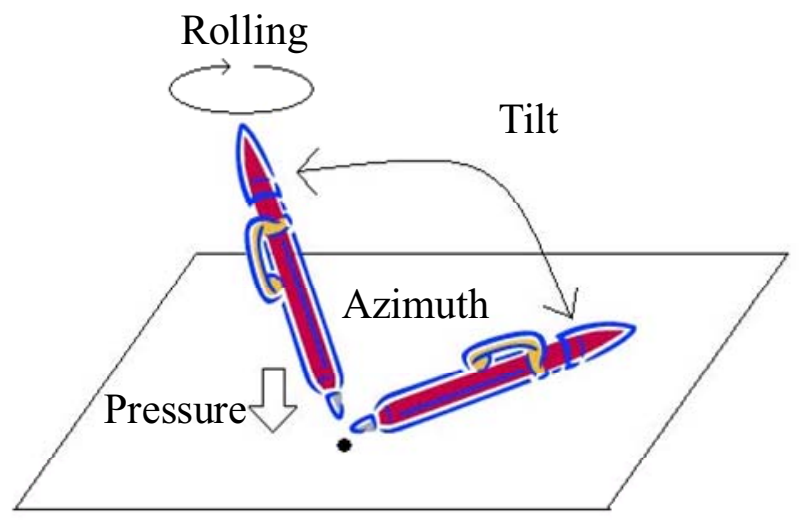

Fig. 1 Inherent input modalities of pen.

In summary, the review indicates that while there are rich literatures on the study of utilization of tablet and pen at the user interface, there has not been an investigation into the effect of the change of environmental parameters, such as tilt angle of tablet, on pen-based input operation. Thus, this is an area that is ripe for research.

\section{EXPERIMENTS}

In order to investigate the effect of tilt angle of tablet on pen-based input operation, we conducted 1-dimensional and 2dimensional pointing experiments based on Fitts' law with 5 kinds of tilt angles of tablet (0, 15, 30, 45 and 60 degrees). From the results of the experiment and questionnaire, we can know how pen-based input operation is effected while tilting the angle of tablet and at which tilt angle of tablet that is easier to do input operation with pen.

\section{A. Experimental Theory}

The experiments in this paper were done based on the widely accepted Fitts' law [11] proposed by Fitts in 1954. Fitts' law predicts that the farther and the narrower the target is, the more time is needed to select the target. Extending Shannon's theorem [16] in information theory, Fitts' law is commonly expressed in the following form [12][17]:

$$
M T=a+b \log _{2}\left(\frac{A}{W}+1\right)
$$

Where MT is the movement time, $\mathrm{A}$ is the amplitude between the centers of two targets, $\mathrm{W}$ is the width of target, $\mathrm{a}$ and $\mathrm{b}$ are empirically determined constants.

The index of difficulty (ID) of the movement is defined as:

$$
I D=\log _{2}\left(\frac{A}{W}+1\right)
$$

Fitts' law is often used as a model for pointing actions in user interfaces, such as interface design and input device evaluation. Further studies of 2-dimensional pointing tasks related to Fitts' law have been done by [13][14][15].

\section{B. Participants}

10 university students, the average age of them was 21.3, participated in the experiments. All of them had normal or corrected normal vision and were right-handed. None of them had formal experience of doing input as a job with pen and tablet.

\section{Apparatus}

The tablet used in the experiments was WACOM Cintiq 21UX interactive LCD graphics display tablet (see Fig.2). The LCD tablet could tilt from 10 degrees to 60 degrees compared with the surface of the table (horizontal) with the adjustment of underplate. The wireless pen used to do input operation came with the LCD tablet. Because 0 degree of the tablet was needed in the experiments and it couldn't be set by adjusting the underplate, we must place a base under the LCD tablet to let the tablet to be 0 degree (horizontal). The experimental software was developed in Java environment and ran on the PC with Windows XP operating system. 


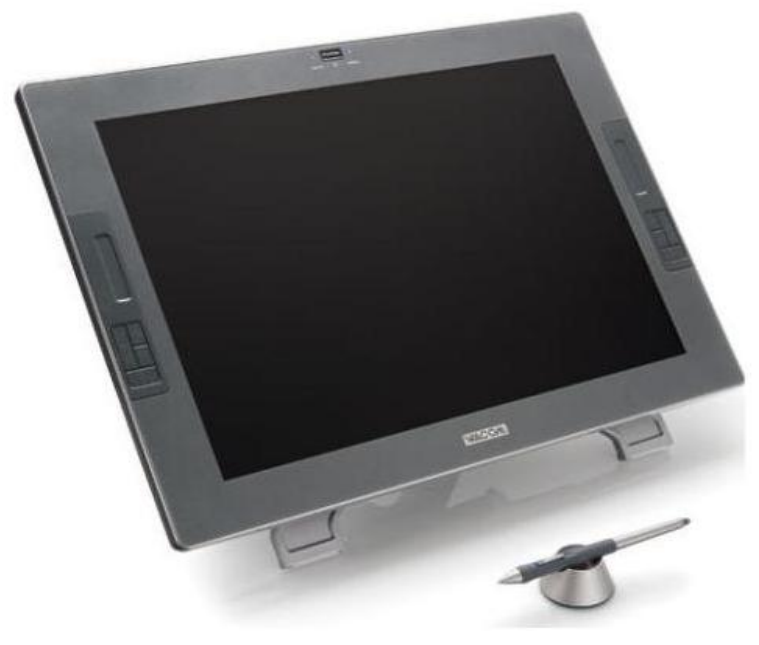

Fig. 2 WACOM Cintiq 21UX.

\section{Design}

The purpose of the experiments was to verify the effect of pen-based input operation caused by the changes of tilt angle of tablet. The result of the preliminary experiments showed that significant differences existed among 0,30 , and 60 degrees of tilt angle of tablet for pen-based input operation. To ensure that meaningful values could be selected, 15 degrees was used as step value in the experiments. Therefore, the tilt angle of tablet from 0 to 60 degrees had 5 levels: 0,15 , 30,45 , and 60 degrees respectively. A protractor, the precision of which was 1 degree, was used to adjust the tilt angle between tablet and the surface of the table (horizontal).

The experiments included 1-dimensional left-right pointing task (linear operation) and 2-dimensional vertical and diagonal pointing task (surface operation).

For 1-dimensional pointing task, 2 vertical strips (see Fig. 3 ) were displayed on the screen, one was white and another was black. The white one was the target needed to be pointed. Once the white one was pointed, the position of the white one and black one were reversed. The participants were required to point the current white one with pen as quickly and accurately as possible in each trial.

In this task, the width (W) of the strips and the amplitude (A) between the center of two strips were set at $\mathrm{W}=20,30,40$ pixels, and $\mathrm{A}=200,300,400$ pixels, and the order of the nine width and amplitude combinations was randomized (see Table I). 10 trials were presented in each $\mathrm{A}$ and $\mathrm{W}$ combination. Each combination was done with five kinds of different tilt angle of tablet $(0,15,30,45$, and 60 degrees) and this was balanced by a Latin Square. In total, the task consisted of:

10 participants $\times$

3 width $x$

3 amplitude $x$

5 tilt angle $\times$

10 repetitions

$=4500$ trials.

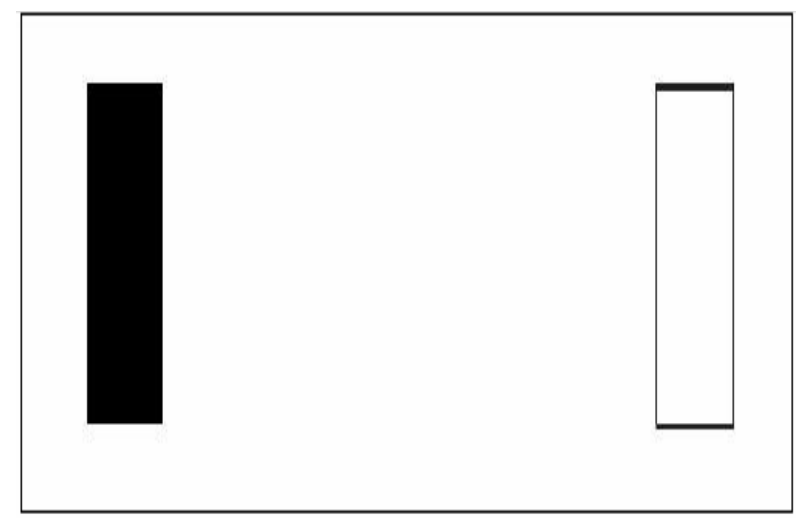

Fig. 3 1-dimensional pointing task.

TABLE I

A-W COMBINATIONS DESIGNED FOR 1-DIMENSIONAL POINTING TASK

\begin{tabular}{|c|c|c|}
\hline Width & Amplitude & ID \\
\hline 20 & 200 & 3.46 \\
\hline 20 & 300 & 4.39 \\
\hline 20 & 400 & 2.94 \\
\hline 30 & 200 & 3.46 \\
\hline 30 & 300 & 3.84 \\
\hline 30 & 400 & 2.58 \\
\hline 40 & 200 & 3.09 \\
\hline 40 & 300 & 3.46 \\
\hline 40 & 400 & \\
\hline
\end{tabular}

For 2-dimensional pointing task, the state of the target on the screen can be seen in Fig. 4. In this task, white circle on the screen was the target needed to be pointed. At the beginning, the white circle appeared in the center of the screen, and once it was pointed, it changed into black. Meanwhile, a white circle in one of the eight compass directions (North, Northeast, East, etc.) around the central circle appeared, and after it was pointed, it disappeared and the central circle became white again to be the next target and so on. Participants were asked to keep on pointing the white one as quickly and accurately as possible in each trial.

In this task, the width (i.e. diameter, $\mathrm{W}$ ) of the targets and the amplitude (A) between the center of two targets were set at $\mathrm{W}=20,30,40$ pixels, and $\mathrm{A}=150,250$ pixels, and the order of the six width and amplitude combinations was randomized (see Table II). The locations of the possibility for the target to appear around the center were eight. Each combination was done with five kinds of different tilt angle of tablet $(0,15,30$, 45 , and 60 degrees) and this was balanced by a Latin Square. In total, the task consisted of:

10 participants $\times$

3 width $\times$

2 amplitude $x$

5 tilt angle $\times$

8 locations

$=2400$ trials. 


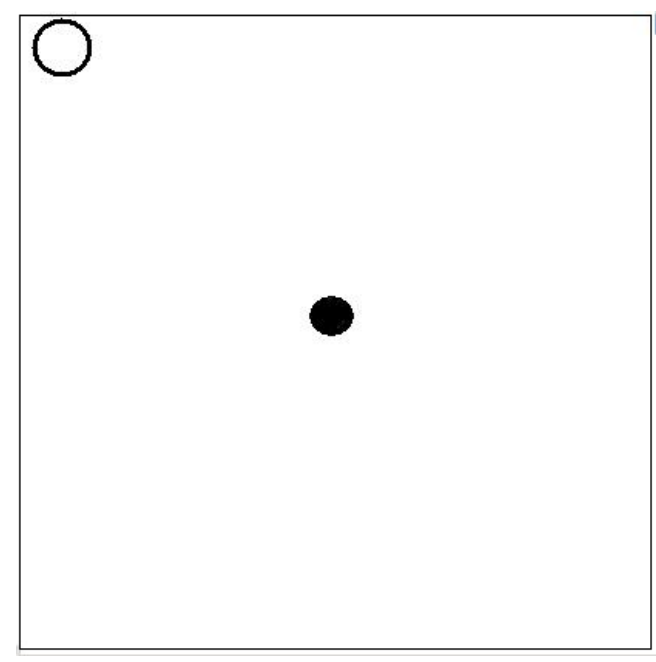

Fig. 4 2-dimensional pointing task.

TABLE II

A-W COMNINATIONS DESIGNED FOR 2-DIMENSIONAL POINTING TASK

\begin{tabular}{|c|c|c|}
\hline Width & Amplitude & ID \\
\hline 20 & 150 & 3.90 \\
\hline 20 & 250 & 4.64 \\
\hline 30 & 150 & 3.32 \\
\hline 30 & 250 & 4.06 \\
\hline 40 & 150 & 2.91 \\
\hline 40 & 250 & 3.64 \\
\hline
\end{tabular}

In each task, after the tilt angle of tablet was set to a certain value, the participant was told to sit down at the table and to adjust the chair to a height suitable for complete the task.

Participants were introduced the whole experimental process and warm-up trials were performed until he/she felt that the experiment could be started.

\section{E. Evaluation Criterion}

Evaluation criterion is acquired from the records of the experiments and the questionnaire of the participants.

Execution time and error rate had been recorded in the experimental records. Execution time referred to the time needed to move from one target to the other. If one pointing dropped out of the target area, an error was recorded and it would not be included in computing execution time.

Participants were asked to rate the fatigue of the hand used and the ease of use of each tilt angle of tablet on questionnaire with seven-point scale ( 1 for worst, and 7 for best) after the experiments.

\section{F. Results}

\section{1) Execution Time}

The Scheffe method was used during the multiple comparisons of experimental results.

From execution time shown in Fig. 5, we could see that for 1-dimensional pointing task there had no significant difference among different tilt angle of tablet. For 2- dimensional pointing task, the tilt angle of 15, 30, 45, and 60 degrees of tablet belonged to the fastest group, the tilt angle of $0,15,45$, and 60 degrees of the tablet belonged to the second fast group, and there was significant difference between 0 and 30 degrees $(\mathrm{p}<.05)$.

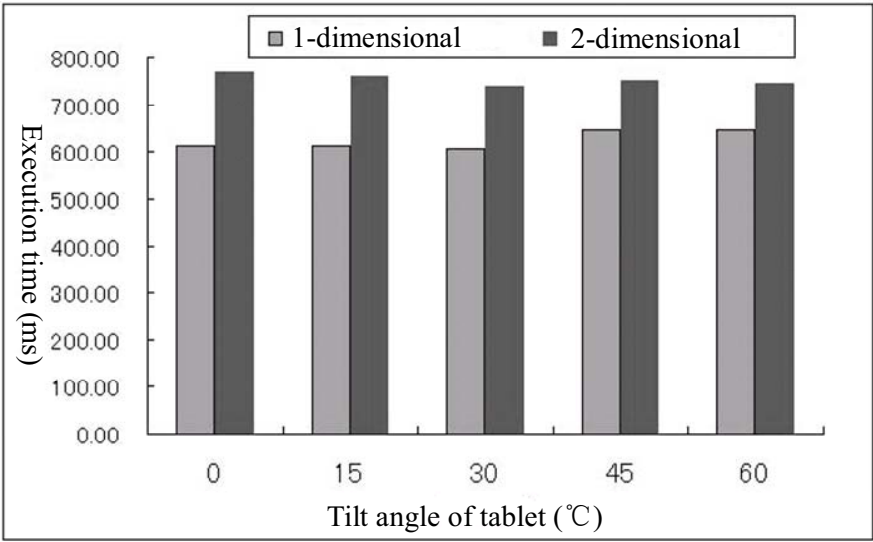

Fig. 5 Execution time of pointing tasks.

\section{2) Error Rate}

The Scheffe method was used during the multiple comparisons of experimental results.

For 1-dimensional and 2-dimensional pointing tasks, the error rates (see Fig. 6) of each tilt angle of tablet belonged to the same group, no significant difference among the tilt angles of tablet.

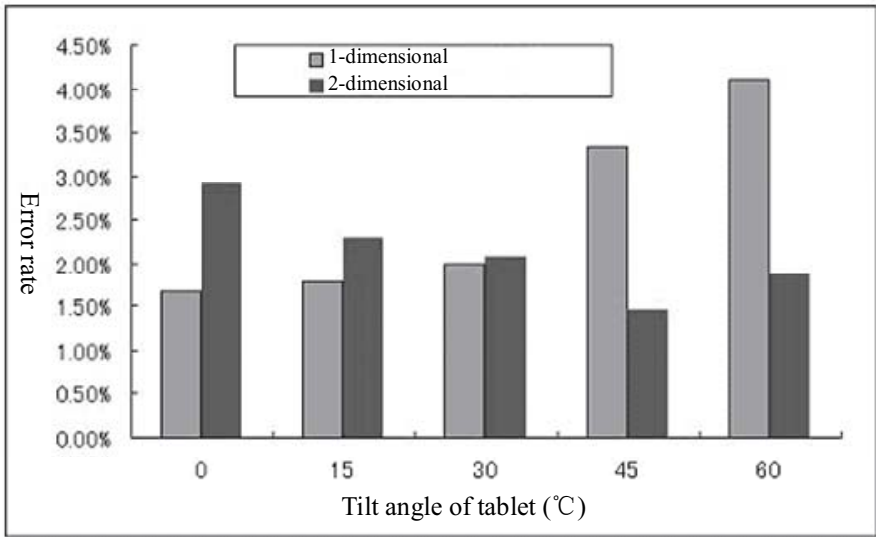

Fig. 6 Error rate of pointing tasks.

\section{3) Subjective Evaluation}

The Tukey-HSD method was used during the multiple comparisons of subjective evaluation.

The subjective evaluation of ease of use (see Fig.7), no matter 1-dimensional pointing task or 2-dimensional pointing task, belonged to the same group for all 5 kinds of tilt angle of tablet, therefore no significant difference between the changes of the tilt angle of tablet.

The subjective evaluation of fatigue (see Fig.8), no matter 1-dimensional pointing task or 2-dimensional pointing task, belonged to the same group for all 5 kinds of tilt angle of 
tablet, therefore no significant difference between the changes of the tilt angle.

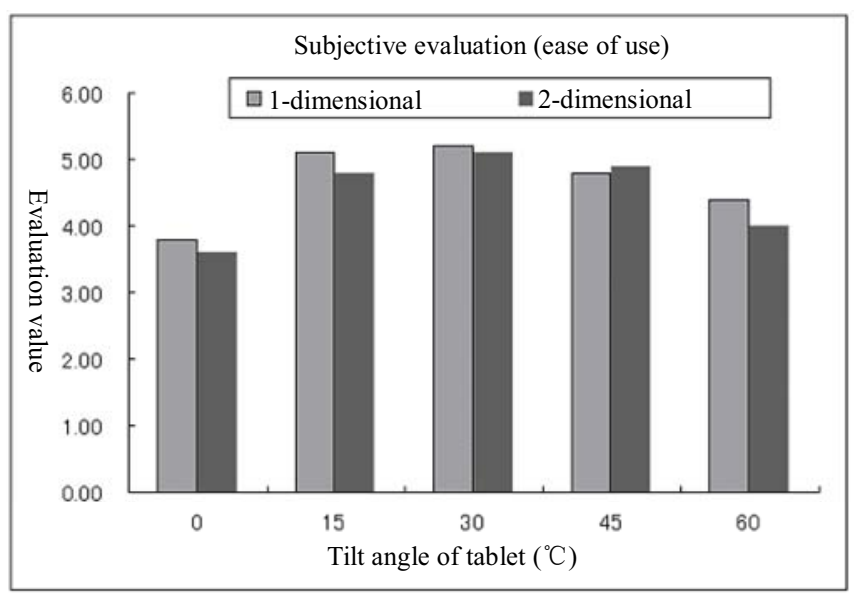

Fig. 7 Ease of use of pointing tasks.

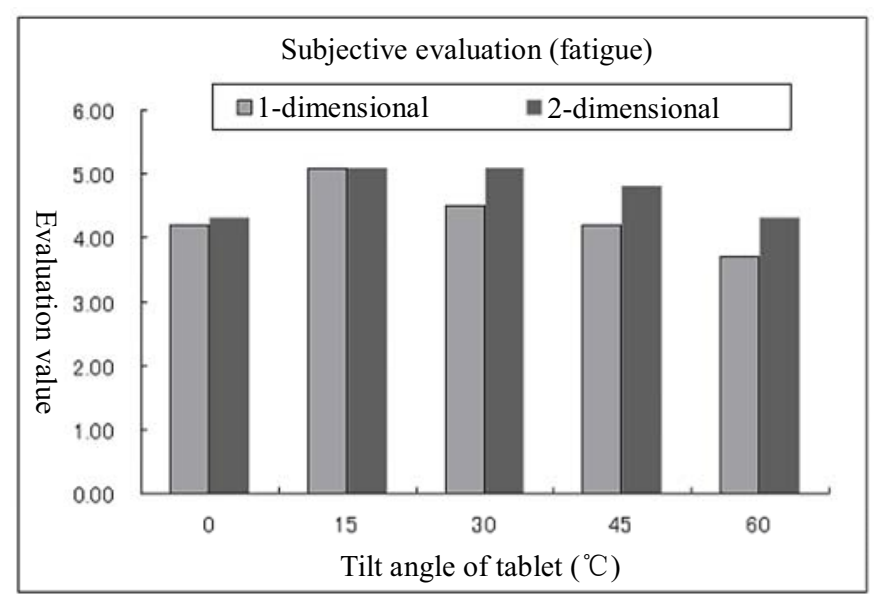

Fig. 8 Fatigue of pointing tasks.

\section{DISCUSSIONS}

\section{A. Execution Time}

Significant difference could be found only in 2dimensional pointing task, the execution time was the shortest when the tilt angle of tablet was 30 degrees, and the execution time was the longest when the tilt angle of tablet was 0 degree.

The execution time was the longest when the tilt angle of tablet was 0 degree in 2-dimensional pointing task. When the tilt angle of tablet was 0 degree (horizontal), the arm was needed to put on the tablet to finish the pointing task, and once the central target was selected, the next target would appear around the center in one of the eight compass directions (North, Northeast, East, etc.). If it resided at the right side of the screen, the target could be blocked by the arm and was hard to be seen, so the time used to choose the target would be longer.

The execution time was the shortest when the tilt angle of tablet was 30 degrees in 2-dimensional pointing task. With the increase of the tile angle of tablet, the need to put the arm on the screen to operate would reduce, and the situations that the target appeared on the right side of the screen that could be blocked by the arm would also reduce. Thereby, the time used to search for the target would shorten.

In 2-dimensional pointing task, the target might appear at the top or the bottom of the screen, and the arm needed to move up and down to select the target. With the increase of the tilt angle of tablet, more vertical movement of the arm was need to point the target, and because of the gravity, vertical movement were hard to be quick and accuracy compared with horizontal movement. If the tilt angle was no so big, the need for the arm to move vertically would reduce, so it was easier to finish the operation with quick and accuracy. As a result, it was thought that when the tilt angle of tablet was 30 degrees, the execution time was the shortest.

\section{B. Error Rate}

For the error rate, no matter 1-dimensional pointing task or 2-dimensional pointing task, there was no significant difference among all 5 kinds of tilt angle of tablet. Although the time used to search the target differed among different tilt angle of tablet, once the target was confirmed, the only thing to do was to select, and the operation itself was not so difficult. Hence, it was thought that the changes of the tilt angle of tablet could not affect the error rate of pointing tasks.

\section{Subjective Evaluation}

For subjective evaluation, no matter 1-dimensional pointing task or 2-dimensional pointing task, there was no significant difference of ease of use and fatigue of hand used among different tilt angle of tablet. Although the operation time changed while changing the tile angle of tablet, the changes were not so dramatic that could be felt by participants. As a result, to subjective evaluation, there was no significant difference while changing the tilt angle of tablet.

\section{CONCLUSIONS AND FUTUREWORKS}

At present, people are trying to use computer anywhere at anytime, and human computer interaction becomes friendlier than ever. Compared to mouse and traditional screen, pen is portable and does not limited by the environment, and touch screen can make input more convenient, accuracy, and quick. These interaction technologies have been broadly used in LCD tablets, tablet PCs, PDAs, and intelligent mobile phones, etc.

Pen-based input operation can be affected by many factors. The tilt angle of tablet was one of the important factor of the operating characteristics of pen-based interface. In this paper, pen was used as direct input device to do the operation at different tilt angles of tablet to verify the influence brought by the execution of the tasks in different experimental environment.

From the results of the experiments we knew 1) for execution time, significant difference existed only in 2dimensional pointing task with the changes of the tilt angle of tablet. The execution time was the shortest when the tilt angle of tablet was 30 degrees. 2) for error rate and subjective evaluation, there was no significant difference among the changes of the tilt angle of tablet. 
To sum up, for pointing tasks, though the changes of the tilt angle of tablet affected execution time, there was no significant differential affection on error rate and subjective evaluation of the participants. Moreover, from the results of pointing tasks, we knew that when the tilt angle of tablet was 30 degrees, it was most suitable for user to do pen-based input operation. The result can be used to make user feel more comfortable in pen-based interface operation and can make the operation more accuracy.

The investigation of this paper has got some conclusions of the operating characteristics of the pen-based interface while the tilt angle of tablet is changed. These conclusions can be the guideline to aid software design to make devices with pen and tablet more ease of use, and it can also be used as a reference of environmental parameter setting in relative experiment designs.

In order to further investigate the effect of tilt angle of tablet on pen-based input operation, we are now doing the experiments of straight and circle stroking tasks based on steering law. With the results from new experiments, we can further verify and certain the conclusions of this paper.

\section{ACKNOWLEDGMENT}

This study was supported by Open Fund of Key Laboratory of Electronics Engineering, College of Heilongjiang Province, (Heilongjiang University), P. R. China (DZZD20100033). This research was done at Ren lab of KUT (Kochi University of Technology). We would like to thank all Ren lab members for their support, ideas and comments, and the anonymous reviewers for their helpful and detailed suggestions about the paper.

\section{REFERENCES}

[1] K. Partridge, S. Chatterjee, V. Sazawal, G. Borriello, and R. Want, "TiltType: Accelerometer-Supported Text Entry for Very Small Devices," ACM UIST Symposium on User Interface Software and Technology, p. 201-204, 2002.

[2] D. Wigdor and R. Balakrishnan, "TiltText: Using Tilt for Text Input to Mobile Phones," ACM UIST Symposium on User Interface Software and Technology, p. 81-90, 2003.

[3] V. Sazawal, R. Want, and G. Borriello. "The Unigesture Approach: OneHanded Text Entry for Small Devices," MobileHCI, p. 256-270, 2002.

[4] J. Rekimoto, "Tilting operations for small screen interfaces," ACM UIST Symposium on User Interface Software and Technology, p. 167-168, 1996.

[5] B. L. Harrison, K. P. Fishkin, A. Gujar, C. Mochon, and R. Want, "Squeeze me, hold me, tilt me! An exploration of manipulative user interfaces," ACM CHI Conference on Human Factors in Computing Systems, p. 17-24, 1998.

[6] G. Levin and P. Yarin. "Bringing sketching tools to keychain computers with an acceleration-based interface," ACM CHI Conference on Human Factors in Computing Systems, p. 268-269, 1999.

[7] G. Ramos, M. Boulos, and R. Balakrishnan, "Pressure Widgets," $A C M$ CHI Conference on Human Factors in Computing Systems, p. 487-494, 2004.

[8] X. Bi, T. Moscovich, G. Ramos, R. Balakrishnan, and K. Hinckley, "An Exploration of Pen Rolling for Pen-based Interaction," ACM UIST Symposium on User Interface Software and Technology, p. 191-200, 2008.

[9] F. Tian, X. Ao, H. Wang, V. Setlur, and G. Dai, "The tilt cursor: enhancing stimulus-response compatibility by providing $3 \mathrm{D}$ orientation cue of pen," ACM CHI Conference on Human Factors in Computing Systems, p. 303-306, 2007.
[10]M. Oshita, "Pen-to-mime: A Pen-Based Interface for Interactive Control of a Human Figure," EUROGRAPHICS Workshop on Sketch-Based Interfaces and Modeling, p. 43-52, 2004.

[11]P. M. Fitts, "The information capacity of the human motor system in controlling the amplitude of movement," Journal of Experimental Psychology, vol. 47, pp. 381-391, 1954.

[12] I. S. MacKenzie, "Fitts' law as a research and design tool in humancomputer interaction," International Journal of Human-Computer Interaction, vol. 7, pp. 91-139, 1992.

[13] I. S. MacKenzie and W. Buxton, "Extending Fitts' law to twodimensional tasks," ACM SIGCHI Conference on Human Factors in Computing Systems, p. 219-226, 1992.

[14] A. Murata, "Extending effective target width in Fitts' law to a twodimensional pointing task," International Journal of Human-Computer Interaction, vol. 11, no. 2, pp. 137-152, 1999.

[15] J. Accot and S. Zhai, "Refining Fitts' law models for bivariate pointing," ACM CHI Conference on Human Factors in Computing Systems, p. 193200, 2003.

[16] C. E. Shannon and W. Weaver, "The mathematical theory of communications," Urbana, Il: University of Illinois Press, 1949.

[17] I. S. MacKenzie, "A note on the information-theoretic basis for Fitts' law," Journal of Motor Behavior, vol. 21, pp.323-330, 1989.

[18] C. Herot, and G. Weinzapfel, "One-point touch input of vector information for computer displays," ACM SIGGRAPH Conference, $\mathrm{p}$. 201-216, 1978.

[19] W. Buxton, et al, "Issues and techniques in touch sensitive tablet input," ACM SIGGRAPH Conference, p. 215-224, 1985.

[20] G. Ramos, and R. Balakrishnan, "Fluid interaction techniques for the control and annotation of digital video," ACM UIST Symposium on User Interface Software and Technology, p. 105-114, 2003.

[21] M. Srinivasan, and J. Chen, "Human performance in controlling normal forces of contact with rigid objects," Proc of ASME, vol. 49, pp. 119-125, 1993.

[22] R. Zeleznik, et al, "Pop through mouse button interactions," ACM UIST Symposium on User Interface Software and Technology, p. 195-196, 2001.

[23] R. Raisamo, "Evaluating different touched-based interaction techniques in a public information kiosk," Conference of the CHI Special Interest Group of the Ergonomics Society of Australia, p. 169-171, 1999. 